Abstract PS1-12

Self-Efficacy and Attendance as Predictors of Weight Loss

Following a Group Intervention

Ronit Endevelt, $\mathrm{PhD}, \mathrm{RD}$, Maccabi Healthcare Services, University of Haifa, Israel; Yael Benyamini, $\mathrm{PhD}$, School of Social Work, Tel Aviv University, Israel; Liora Valinsky, MPH, RN Maccabi Healthcare Services, School of Public Health, Tel Aviv University, Israel; Rivki Geron, MSw, School of Social Work, Tel Aviv University, Israel; Nurit Medini, MSw, School of Social Work, Tel Aviv University, Israel

Background: Self-efficacy in a specific area has typically been shown to predict actual success in goal attainment. However, in the dieting area there have been inconsistent findings regarding the relationship between self-efficacy and weight loss. Therefore, the main aims of the current study were (a) to test the success of a short-term group intervention in achieving weight loss, (b) to test whether participation in the intervention improves self-efficacy, (c) to test whether eating-related self-efficacy predicts weight loss in this program, and (d) to examine whether attendance in more sessions of the program is related to increased weight loss. Methods: The study followed up on a group intervention aimed at lifestyle changes, which took place at the Maccabi HMO and was facilitated by trained dietitians. The group method was based on nutritional skills coaching (e.g., planning a balanced diet, incorporating behavior and lifestyle changes). There were 190 participants in 19 groups of a 10-session program who took part in the study. Participant mean age was 45 years $(\mathrm{SD}=12 \mathrm{yrs}), 92 \%$ were female and $60 \%$ had post-high school education. Participants filled in the Eating Self-Efficacy Scale at the second and last sessions, and provided self-reported weight by phone 3-months and 12-months post-intervention. Group facilitators measured height and weight, and noted attendance at each session. Results: BMI significantly decreased from the first session (mean $\mathrm{BMI}=31.4$ ) to the last one (BMI 30.4). Weight loss (calculated as change in $\mathrm{BMI})$ was retained; further non-significant decreases were seen at 3-months $(B M I=30.1)$ and 12-months $(B M I=29.8)$. Participants attended an average of 7.5 sessions and increased attendance was correlated with greater weight loss $(\mathrm{r}=0.20, P<0.05)$. Eating self-efficacy (in emotional states and social situations) significantly improved during the course of the group intervention. Initial low emotions-related self-efficacy was related to higher initial BMI but only initial low social-situations-related self-efficacy was related to weight loss. Higher goals at program outset were linearly related to greater weight loss. Conclusions: The short-term group intervention was successful in attaining weight loss and improving self efficacy. Participants who initially had difficulties in controlling overeating in social situations benefited the most from the program. Attendance in more sessions of the program was found to be related to increased weight loss.

\section{Abstract PS1-13}

High Five for Kids: Predictors of Participation in a Clinically-Based Obesity Prevention Intervention

Katherine H. Hohman, MPH, Department of Ambulatory Care and Prevention; Sarah N. Price, MPH, Department of Ambulatory Care and Prevention; Sheryl L. Rifas-Shiman, MPH, Department of Ambulatory Care and Prevention; Elsie M. Taveras, MD, MPH, Department of Ambulatory Care and Prevention; Matthew W. Gillman, MD, SM, Department of Ambulatory Care and Prevention

Aim: To examine predictors of participation in an obesity prevention trial among preschool-age children. Method: High Five for Kids is a cluster-randomized controlled trial of a planned 500 children in 10 primary care pediatric offices. Eligible children are 2-6.9 years old with BMI $>95^{\text {th }}$ percentile (obese), or $85^{\text {th }}-95^{\text {th }}$ percentile (overweight) if at least one parent is overweight. The initial intervention period is 12 months, with a 1 -year follow-up period, and the primary outcome is BMI. Through screening of electronic medical records, we have identified and contacted 766 age-eligible and BMI-eligible children to date. We recruit parents via telephone following an introductory letter. We interviewed 220 of $306(72 \%)$ parents who refused participation (refusers). Results: Parents who refused participation $(\mathrm{n}=220)$ did not differ from those who participated $(\mathrm{n}=470)$ by education $(62.8 \%$ vs $55.3 \%$ with college degree, $P=0.07$ ) or by the number of children at home ( 2.2 vs $2.3, P=0.21$ ). Children of parents who refused participation did not differ from those who participated by age ( 4.5 yrs vs 4.6 yrs, $P=0.65$ ) or sex
( $48.1 \%$ vs $44.5 \%$ female, $P=0.36$ ). The two groups did differ by proportion of children in BMI category ( $63.6 \%$ vs $52.9 \%$ overweight and $36.4 \%$ vs $47.0 \%$ obese, $P=0.009)$. After adjusting for parent education level, number of children at home, and child age and sex, we found that parents were $53 \%$ more likely $(\mathrm{OR}=1.53 ; 95 \% \mathrm{CI}, 1.10-2.14)$ to participate if their children were obese vs. overweight. Among the 80 refusers with obese children, $27.5 \%$ cited as a reason for refusal that their children did not have a problem with weight versus $37.9 \%$ among the 140 refusers with overweight children. Conclusions: Overweight parents of preschool-age children with a BMI $85^{\text {th }}-95^{\text {th }}$ percentile are less likely to have their children participate in an obesity prevention trial than parents of children with $\mathrm{BMI}>95^{\text {th }}$ percentile. One reason appears to be that they less frequently consider their children to have a weight problem.

\section{Abstract PS1-15 \\ Assessment of First-Fill Adherence Rates For Patients With Hypertension Using Linked EHR and Claims Data}

Nirav R. Shah, MD, MPH, Geisinger Center for Health Research; Annemarie G. Hirsch, MPH, Geisinger Center for Health Research; G. Craig Wood, MS, Geisinger Center for Health Research; Amanda F. Wingate, BA, Geisinger Center for Health Research; Ilene G. Ladd, MS, Geisinger Center for Health Research; Walter F. Stewart, PhD, MPH, Geisinger Center for Health Research

Background: Much of the distance between the promise of evidence-based medicine and reality of improved patient outcomes can be attributed to problems in the 'last mile,' or patient adherence - the extent to which a person's behavior coincides with medical or health advice. Studies of prescription adherence have been hampered by methodologic problems. Most rely on patient self-report of adherence or cross-sectional data of plan-wide prescription fills to estimate patient-level adherence. We used a unique combination of electronic health record (EHR) data from clinics and prescription claims data to understand first-fill rates for hypertension and factors associated with first-fill behavior. Methods: We conducted a retrospective cohort study and linked individual patient data for new (i.e., first-time) prescriptions of medications for hypertension from EHR, with claims data obtained from an insurance plan. EHR data were obtained from the Geisinger Clinic, a large group practice serving a 31-county area of central and northeastern Pennsylvania. The Clinic includes 41 community practice sites with primary and multi-specialty care, all of which have used an EHR since 2001. Adherence was defined as a claim generated for the first-fill prescription within 30 days of the index date. Results: Of 3240 patients written a new, first-time prescription for any antihypertensive medication, 2685 (83\%) generated a corresponding claim within 30 days. Gender, age, therapeutic class, number of medications prescribed within 10 days of prescription, number of refills, co-pay, comorbidity score, baseline systolic blood pressure, baseline diastolic blood pressure, change in systolic blood pressure, and change in diastolic pressure were significantly associated with first-fill rates $(P<0.05)$. Race, number of hypertension medications ordered, number of office visits prior to prescription, post-systolic blood pressure, and post-diastolic blood pressure had no association. Conclusions: The data reveal characteristics of patients who are less likely to adhere to treatment for hypertension. Patients who are older, female, have comorbidities, and/or have relatively lower blood pressures may be less likely to adhere to treatment for hypertension. Patients with these characteristics may be potential candidates for interventions to improve adherence.

\section{Abstract PS1-16 \\ A Study Design to Evaluate Group Dialogue Activities to Improve Diabetes Outcomes}

JoAnn M. Sperl-Hillen, MD, HealthPartners Research Foundation; Sarah J. Beaton, PhD, Lovelace Clinic Foundation; Adrine Chung, HealthPartners Research Foundation; Ann Von Worley, RN, BSHS, Lovelace Clinic Foundation; Jodi Lavin-Tompkins, RN, CDE, HealthPartners Research Foundation

Background: Behavior change and self-efficacy are key for patients with diabetes to sustain blood sugar goals, but many do not receive enough support for successful ongoing self-management. After initial education, Medicare reimbursement for diabetes education, commonly delivered on an 